Cinémas

Revue d'études cinématographiques

Journal of Film Studies

\title{
La Salle de bain : l'immobilité cinétique
}

\section{Jean-Claude Sommier}

Volume 4, numéro 1, automne 1993

\section{Écrit/Écran}

URI : https://id.erudit.org/iderudit/1000114ar

DOI : https://doi.org/10.7202/1000114ar

Aller au sommaire du numéro

Éditeur(s)

Cinémas

ISSN

1181-6945 (imprimé)

1705-6500 (numérique)

Découvrir la revue

Citer cet article

Sommier, J.-C. (1993). La Salle de bain : l'immobilité cinétique. Cinémas, 4(1),

103-114. https://doi.org/10.7202/1000114ar

\section{Résumé de l'article}

L'étude de cas suivante, la comparaison entre le roman La Salle de bain et son adaptation cinématographique, tente de faire la part de l'autonomie et de la dépendance du film vis-à-vis du roman. Deux problématiques sont en jeu : une, de type pascalien, de l'individu, et une seconde, articulée sur la précédente, du rapport temps/mouvement. Il semble que dans le roman s'opère un glissement de l'une à l'autre pour privilégier la seconde alors que le film paraît privilégier la première. L'auteur explicite cette différence à la fois par la nature du médium et en terme d'instance(s) narratrice(s) : passage du roman en «je» au «il» imposé par la caméra. 


\title{
La Salle de bain : l'immobilité cinétique
}

\section{Jean-Claude Sommier}

\begin{abstract}
RÉSUMÉ
L'étude de cas suivante, la comparaison entre le roman La Salle de bain et son adaptation cinématographique, tente de faire la part de l'autonomie et de la dépendance du film vis-à-vis du roman. Deux problématiques sont en jeu : une, de type pascalien, de l'individu, et une seconde, articulée sur la précédente, du rapport temps / mouvement. Il semble que dans le roman s'opère un glissement de l'une à l'autre pour privilégier la seconde alors que le film paraît privilégier la première. L'auteur explicite cette différence à la fois par la nature du médium et en terme d'instance(s) narratrice(s) : passage du roman en «je» au «il» imposé par la caméra.
\end{abstract}

\begin{abstract}
This case study comparing the novel La Salle de bain and its film adaptation attempts to set out both the autonomy and the dependence of the film vis-à-vis the novel. Two problematics are considered : one, Pascalian, of the individual, and the second, articulated on the first, of the time/movement relationship. It appears that in the novel there is slippage from one to the other in order to privilege the second, while the film seems to privilege the first. The author explains this difference both by the nature of the medium and in terms of narrative instance(s) : the passage from the "I" of the novel to the "he" imposed by the cinema.
\end{abstract}

Cette étude se donne pour objet la comparaison du roman de Jean-Philippe Toussaint, La Salle de bain, à son adaptation 
cinématographique du même titre par John Lvoff (1989). Voici les définitions que donnent de «bain» et de «salle de bains» le Grand Larousse de la langue française :

Bain : 1) Action d'immerger le corps ou une partie du corps dans un liquide, un gaz, à des fins hygiéniques ou thérapeutiques; 2) Fig. Contact prolongé avec une œuvre, un milieu, une pensée : «Pourquoi y-a-t-il si peu de gens qui aient l'idée de prendre des bains de silence ?» - Claudel (Tome I, p. 358).

Salle de bains : pièce aménagée pour les soins corporels et comprenant une baignoire, un lavabo, un bidet et éventuellement une douche (Tome VI, p. 5338).

Cette dernière définition traduit une organisation très stricte du réel, que nous retrouvons dans le comportement initial du personnage principal du film.

Voici, pour brièvement relater (frelater) l'histoire ${ }^{1}$, un montage des critiques parues dans Le Canard enchaîné du mercredi 8 mars 1989 («De la plus belle eau», p. 6) et dans Le Monde du dimanche 12-lundi 13 mars 1989 («Visages pâles, p. 16), parfois complété de citations du roman. Ces critiques ne me semblent intéressantes que par leur mimétisme avec l'œuvre en question (qui tient du minimalisme) :

«Le roman de Jean-Philippe Toussaint, La Salle de bain, connut un beau succès en 1985, on loua son économie, sa brièveté, sa sécheresse ironique, ce plaisir de faire court sans faire cours qui est une des facettes du génie français»( $L e$ Monde). "Jean-Philippe Toussaint est belge» (Le Canard enchaîné). "On ne pouvait pas imaginer plus discret, ce fut donc une petite bombe. Un exemple de sobriété postmoderne, un manifeste minimaliste» (Le Monde). "La critique pousse des "oh!" d'admiration, les lecteurs suivent. Aujourd'hui le film. Réalisé par John Lvoff» (Le Canard enchaîné), «(...) ancien assistant de Resnais, de Rappeneau, de Polanski» (Le Monde). «Avec adaptation et dialogues de l'auteur en personne»( $L e$ Canard enchaîné).

Un jeune homme, âgé de «27 ans, bientôt 29» (p.15), médite dans sa baignoire où il «coul[e] là des heures agréables» (p.11). Il décide de prendre «le risque de compromettre la quiétude de [s]a vie abstraite» (p.15) et sort de la salle de bain pour affronter un monde qui s'agite bien trop à son gré. «Il observe. Intervient par des "je ne sais pas" et des "peut-être". Puis s'en va. Il est à Venise. Dans un hôtel à moitié vide. Il joue aux fléchettes dans sa chambre» (Le Canard enchaîné). Sa compagne (qui se nomme ou se prénomme ? - Edmondsson) le rejoint. "Il lui colle une fléchette en plein front, elle repart. C'est lui qui va se faire 
radiographier le crâne, ensuite, pour sinusite. Puis sympathiser avec le docteur et dîner chez ce dernier, d'un plat de rognons, avant de rentrer à Paris» (Le Monde). Où il retrouve son appartement et ses habitudes, c'est-à-dire sa salle de bain, pendant quelque temps et un jour décide de prendre «le risque de compromettre la quiétude de [s]a vie abstraite» (p.123) et sort de la salle de bain. Voilà, succintement résumé, ce qui se passe dans le roman et le film.

Résumé truqué, faux, qui tente de faire accroire la possibilité d'un va-et-vient trans-sémiotique entre le récit écrit et le récit filmique. Le roman deviendrait ainsi le scénario ou - pire - le script du film. Mais je reviendrai plus loin sur ce problème.

Second point de départ de ce travail : trois citations des Pensées de Pascal:

131 - Ennui. - Rien n'est si insupportable à l'homme que d'être dans un plein repos, sans passions, sans affaires, sans divertissement, sans application. Il sent alors tout son néant, son abandon, son insuffisance, sa dépendance, son impuissance, son vide. Incontinent il sortira du fond de son âme l'ennui, la noirceur, la tristesse, le chagrin, le dépit, le désespoir (p. 85).

135 - Rien ne nous plaît que le combat, mais non pas la victoire (...). Nous ne cherchons jamais les choses, mais la recherche des choses (p. 86).

139 - Divertissement. - Quand je m'y suis mis quelquefois à considérer les diverses agitations des hommes et les périls et les peines où ils s'exposent, dans la cour, dans la guerre, d'où naissent tant de querelles, de passions, d'entreprises hardies et souvent mauvaises, etc., j'ai découvert que le malheur des hommes vient d'une seule chose, qui est de ne savoir pas demeurer en repos, dans une chambre. (...) Mais quand j'ai pensé de plus près, et qu'après avoir trouvé la cause de tous nos malheurs, j'ai voulu en découvrir la raison, j'ai trouvé qu'il y en a une bien effective, qui consiste dans le malheur naturel de notre condition faible et mortelle, et si misérable, que rien ne peut nous consoler, lorsque nous y pensons de près. (...) De là vient que le jeu et la conversation des femmes, la guerre, les grands emplois sont si recherchés. Ce n'est pas qu'il y ait en effet du bonheur, ni qu'on s'imagine que la vraie béatitude soit d'avoir l'argent qu'on peut gagner au jeu, ou dans le lièvre qu'on court : on n'en voudrait pas s'il était offert. Ce n'est pas dans cet usage mol et paisible, et qui nous laisse penser à notre malheureuse condition, qu'on recherche, ni les dangers de la guerre, ni la peine des emplois, mais c'est le tracas qui nous détourne d'y penser et nous divertit (pp. 86-87, c'est moi qui souligne).

Le résumé fait au début de cet article est insatisfaisant, car il postule qu'entre le roman et le film n'existe qu'une différence de médium et que le réalisateur, aidé en cela de l'auteur scénariste, 
est parvenu à une adéquation de l'écrit à l'écran. En effet, si je suis un spectateur naïf, a priori, les différences entre le roman et son adaptation cinématographique sont minimes et ne concernent que des détails d'ordre diégétique. (Et je pense que l'on peut parler de détails dans le cas présent parce que la diégèse est réduite à peu de chose. En d'autres termes, si je fais un synopsis du roman et un synopsis du film, j'arrive à la même chose, à savoir le résumé donné plus haut.)

Mais en tant que spectateur qui a lu Genette et qui a écouté François $\mathrm{Jost}^{2}$, je me suppose donc un peu plus averti. Alors, si je perçois des différences entre le récit écrit et le récit filmique ${ }^{3}$, elles seront au niveau de la narration.

Mon étude s'articule ainsi autour de l'instance narratrice : une instance narratrice unique, un «je» - sans ambiguïté - qui s'exprime au passé dans le roman et une instance narratrice double dans le film : la caméra et le sujet anonyme (appelons-le ici le personnage principal), qui se meuvent dans un présent de représentation (c'est une présentation parce qu'il s'agit d'une narration qui s'actualise au présent, devant moi spectateur. Et c'est une représentation parce qu'il s'agit de déjà-filmé. Je suis conscient de ce que cette formulation a d'insatisfaisant, mais je vais y revenir plus loin).

Dans ce roman à la première personne qu'est La Salle de bain, toute la narration se fait en focalisation interne fixe (Genette, p. 206), qui fait appréhender les situations à travers la conscience du «je» narrateur autodiégétique (Genette, p. 253).

«Je» s'enferme dans sa salle de bain, seul lieu où il est bien et où il médite, satisfaisant ainsi à l'exigence pascalienne du repos. La salle de bain est le lieu du repli sur soi, loin des distractions, des diversions :

Je répondis [à ma mère] que le besoin de divertissement me paraissait suspect. Lorsque, en souriant presque, j'ajoutai que je ne craignais rien moins que les diversions, elle vit bien que l'on ne pouvait pas discuter avec moi (p. 13).

Également lieu de la non-agitation, du non-mouvement, de l'immobilité :

Autour de moi se trouvaient des placards, des porte-serviettes, un bidet. Le lavabo était blanc; une tablette le surplombait, sur laquelle reposaient brosses à dents et rasoirs. Le mur qui me faisait face, parsemé de grumeaux, présentait des craquelures; des cratères çà et là trouaient la peinture terne. Une fissure semblait gagner du terrain. Pendant des heures, je guettais ses extrémités, essayant vainement de surprendre un progrès. Parfois, je tentais d'autres expériences. Je surveillais la surface de mon visage dans un miroir de poche et parallèlement, les déplacements de l'aiguille 
de ma montre. Mais mon visage ne laissait rien paraitre. Jamais (p. 12).

«Je» est un sujet que je qualifierais de schizothymique, c'est-àdire qu'il a «une faible affectivité et une tendance à l'intériorité, mais sans trouble psychique» (Grand Larousse de la langue française, T. VI).

Sujet excentrique, excentré, ou qui se veut tel par rapport au monde qui l'entoure, mais toujours au centre, ne serait-ce que par les conditions mêmes de la narration : il est le sujet de l'énonciation, donc par définition celui vers qui le regard se tourne ${ }^{4}$. «Je» est, ce me semble, au cœur d'une double problématique. D'une part une problématique de l'individu, de type pascalien, où «je» choisit l'isolement, s'enferme dans cette pièce "pour y demeurer en repos», sans le trouver. D'autre part, articulée sur la précédente, une problématique du rapport temps/mouvement. Et un glissement s'opère de l'une à l'autre, privilégiant, à mon avis, la seconde. Sur le plan thématique, «je» parle de l'écoulement du temps qui l'horrifie :

Assis sur mon lit, la tête dans les mains (toujours ces positions extrêmes), je me disais que les gens ne redoutaient pas la pluie; (...) nul n'avait vraiment peur qu'elle ne s'arrêtât plus jamais, écoulement continu faisant tout disparaître - abolissant tout. C'est moi qui (...) avais eu soudain peur du mauvais temps, alors que c'était l'écoulement du temps, une fois de plus, qui m'avait horrifié (p. 31)5.

Ou :

J'avais passé la nuit dans un compartiment de train, seul, la lumière éteinte. Immobile. Sensible au mouvement, uniquement au mouvement, au mouvement extérieur, manifeste, qui me déplaçait malgré mon immobilité, mais aussi au mouvement intérieur de mon corps qui se détruisait, mouvement imperceptible auquel je commençais à vouer une attention exclusive, qu'à toutes forces je voulais fixer. Mais comment le saisir ?6 Où le constater? Les gestes les plus simples détournèrent l'attention. Je tendis mon passeport vert à un policier italien (p. 54)7.

\section{Ou encore :}

Je regardais la dame blanche fondre devant moi. Je regardais fondre imperceptiblement la vanille sous la nappe de chocolat brûlant. (...) Je regardais le mouvement, immobile, les yeux fixés sur la soucoupe. Je ne bougeais pas. Les mains figées sur la table, j'essayais de toutes mes forces de garder l'immobilité, de la retenir, mais je sentais bien que, sur mon corps aussi, le mouvement s'écoulait (p. 80) 8 . 


\section{Et surtout :}

Ce qui me plaît dans la peinture de Mondrian, c'est son immobilité. Aucun peintre n'a voisiné d'aussi près l'immobilité. L'immobilité n'est pas l'absence de mouvement, mais l'absence de toute perspective de mouvement, elle est mort. La peinture, en général, n'est jamais immobile. Comme aux échecs, son immobilité est dynamique. Chaque pièce, puissance immobile, est un mouvement en puissance. Chez Mondrian, l'immobilité est immobile (...) (p. 84)9.

Si la recherche de l'immobilité est ce à propos de quoi le sujet écrit, cette recherche s'accompagne de pratique. D'une part dans le monde où se meut le sujet :

Je quittai l'hôtel et, dans la rue, demandai le chemin de la poste à un homme qui courait (j'ai toujours pris plaisir à demander des renseignements à des gens pressés). Il m'indiqua rapidement une direction du doigt et voulut m'éviter pour continuer sa route, mais, lui bloquant courtoisement le passage, je lui demandai quelques éclaircissements. À ce moment-là, il s'immobilisa vraiment et, prenant la peine de se retourner, avec beaucoup de patience, me donna toutes les indications nécessaires (pp. 63-64).

D'autre part dans l'espace même de la narration : par le phénomène de dislocation, c'est-à-dire le rejet en fin de proposition de la subordonnée, «normalement» incise. Exemple :

Je fus très amical, souriant, expliquant que j'avais besoin de la salle de bain, mais que l'évier était à sa disposition, dans lequel reposaient ses calmars (p. 24).

Traditionnellement (quand nous nous référons à nos années de lycée), ceci est considéré comme stylistiquement mauvais. Je vois cela comme un moyen de ralentir la séquence de narration, dans la mesure où, par habitude culturelle, nous tentons de restructurer la phrase selon le modèle canonique appris, c'est-àdire avec incise : «l'évier, dans lequel reposaient ses calmars, était à sa disposition». En récrivant la proposition, nous freinons la lecture. Tout comme le narrateur essaie de freiner, à défaut d'arrêter, le temps.

Il reste à mentionner la présentation du roman et son humour. Le roman est divisé en trois parties (Paris, l'hypothénuse, Paris), divisées respectivement en 40,80 et 50 "paragraphes» (ou chapitres ou pensées). Chaque paragraphe représente une pensée, une remarque, quelques phrases et n'a pas forcément de rapport évident de continuité temporelle, thématique ou autre avec ce qui précède et/ou ce qui suit. Ce moyen formel permet de syncoper, de couper la narration, et ainsi de ralentir, voire de stopper la 
diégèse. Le roman se termine par deux paragraphes qui reprennent terme à terme les paragraphes 10 et 11 de la première partie. L'écriture-lecture ne pouvant atteindre l'immobilité, elle peut au moins recommencer son cycle, déjouant ainsi l'entropie. Nous refaisons au niveau du roman ce que nous faisions lors des dislocations.

En ce qui concerne l'humour, concept à manipuler avec précaution, il me paraît jouer un double rôle de mise à distance, d'une part entre le narrateur et le narrataire, et, d'autre part, entre le sujet de l'énonciation et son énoncé; ou, pour introduire le topos de la schizophrénie ${ }^{10}$ qui paraît à la fin du film, entre le sujet de l'énonciation et le sujet de l'énoncé qui serait dans ce cas le même et un autre. Je ne prendrai qu'un seul exemple, la litote :

Peu à peu, je commençais à sympathiser avec le barman. (...) Lorsque j'allais prendre mon café, en fin d'après-midi, il nous arrivait de converser. (...) L'absence d'une langue commune ne nous décourageait pas; sur le cyclisme, par exemple, nous étions intarissables. Moser, disait-il. Merckx, faisais-je remarquer au bout d'un petit moment. Coppi, disait-il, Fausto Coppi. Je tournais ma cuillère dans le café, approuvant de la tête, pensif. Bruyère, murmurais-je. Bruyère? disait-il. Oui, oui, Bruyère. Il ne semblait pas convaincu. Je pensais que la conversation s'en tiendrait là, mais alors que je me disposais à quitter le comptoir, me retenant par le bras, il m'a dit Gimondi. Van Springel, répondis-je. Planckaert, ajoutai-je, Dierieckx, Willems, Van Impe, Van Looy, de Vlaeminck, Roger de Vlaeminck et son frère, Eric. Que pouvait-on répondre à cela ? Il n'insista pas. Je payai le café et remontai dans ma chambre (pp. 61- 62).

Alors que des deux problématiques, celle de l'individu et celle de la relation temps/mouvement, le roman me semble privilégier la seconde, le film me paraît privilégier la première.

Je vais tenter d'expliciter cette différence entre le roman et son adaptation cinématographique par la nature du médium.

Dans le récit filmique, il n'est plus question de narrateur autodiégétique. Nous nous trouvons face à une double instance narrative, celle de la caméra et celle de celui qui, tout à l'heure, se nommait «je» et qui est devenu «il» (d'autant plus «il» que l'on ignore tout de son identité11). Si je décide d'adopter le terme de méganarrateur (Gaudreault et Jost, p. 56) pour qualifier l'instance narrative de la caméra, je vais garder, pour des raisons pratiques, celui de «sujet» pour le second, afin de garder le terme utilisé plus haut, si je fais à nouveau référence au roman. 
Qui raconte ? La caméra, ou méganarrateur. Qui est raconté ? Celui que, tout à l'heure (dans le roman), je ne pouvais pas voir, mais qui m'est désormais montré (dans le film).

Voyons quelques paramètres qui ont changé. Dans le roman, la narration était appréhendée par le sujet. Dans le film, il n'y a que trois cas de focalisation interne : une prolepse, quand «il» devine ce qui va se passer chez l'ambassadeur d'Autriche, et deux analepses, quand «il» se rappelle la visite aux anciens locataires et quand «il» se souvient du jour où sa compagne et lui ont pendu la crémaillère.

Le sujet ressemble, tout le monde le remarque, à Buster Keaton, c'est-à-dire le symbole même de l'impassibilité. On a accentué son maquillage, qui n'est pas sans rappeler le temps du muet et qui, graphiquement, renforce son isolement.

Le film a bien tenté de garder la structure syncopée du livre en trouvant une équivalence des paragraphes sous la forme de tableaux. Mais, rapidement, la caméra devient un tant soit peu plus souple et le film perd ce fractionnement. Nous avons moins de plans champ contrechamp qui permettent d'isoler le personnage du reste du monde (lors de la visite de sa mère, la conversation est montrée de cette manière, et l'isolement du personnage est accentué par le fait qu'il reste allongé dans la baignoire dont le bord le sépare de sa mère assise sur le bidet).

Cela tient au fait que, peu à peu, la diégèse l'emporte. Et cela tient à la nature du médium. Dans un livre, si fractionné soit-il, j'ai toujours la possibilité de m'arrêter et de relier entre eux les éléments que j'estime pertinent de voir reliés. Mais la vitesse de défilement du film et surtout son impossibilité de revenir en arrière, ou de faire arrêt sur image (je parle, bien entendu, d'un visionnement en salle de cinéma et non de vidéo) font que le spectateur a moins d'éléments auxquels il peut se raccrocher. Ainsi nous connaissons deux moyens d'aider le spectateur à suivre ce que lui raconte le méganarrateur : l'un est le renforcement de la structure diégétique au niveau de la narration (on donne plus de poids à la diégèse), l'autre est la mise en place de paradigmes, donc d'images récurrentes qui peu à peu révèlent une signification (cf.: la récurrence des images montrant le fascisme et l'homophobie de la société anglaise thatchérienne dans The Last of England de Derek Jarman). C'est ce dernier moyen que nous avons à l'œuvre dans La Salle de bain, me semble-t-il.

Je signalais plus haut que le film privilégiait la problématique du personnage par rapport à celle de la relation mouvement/temps (privilégiée par le roman). C'est à partir d'un 
sème polysémique que se construit la divergence. Lorsque le sujet regarde en même temps son visage dans un miroir tenu obliquement et sa montre, nous avons à la fois mise à distance du sujet par lui-même et la problématique du temps liée au mouvement ${ }^{12}$. Dans le film sera développée l'isotopie du miroir qui prend une autonomie diégétique absente du roman.

Ainsi, après son installation dans une chambre d'hôtel de Venise, le personnage se met-il à attendre les appels téléphoniques de sa compagne. Lors de ces appels, il transporte l'appareil et le combiné près d'un miroir devant lequel il s'accroupit, ou se recroqueville, en tournant de plus en plus le dos au miroir, donc à sa propre image. Il en va de même dans sa chambre où il joue aux fléchettes. Il a fixé la cible à l'une des deux portes de l'armoire (la gauche pour le spectateur), l'autre (la droite) portant un miroir sur toute sa hauteur. Lorsqu'il s'entraîne, la caméra est placée de trois quarts arrière gauche : le spectateur ne peut donc voir que la cible et le miroir (et le personnage, bien sûr, cadré en plan américain). Peu avant qu'il ne lance une fléchette dans le front de son amie, la caméra, qui nous le montre lors de son entraînement, est passée à sa droite; le spectateur voit donc le personnage de trois-quart arrière droit, la cible, le miroir et le reflet du personnage dans le miroir : le personnage semble donc lancer les flèches à son image. C'est luimême qu'il vise et dont il cherche à atteindre le centre. Le dernier plan qui le montre occupé à cette activité est un plan rapproché : la distance qui le sépare de lui-même diminue, la tension monte, et même, pour la première fois, son visage perd son impassibilité pour trahir une certaine dureté.

Si l'épisode de la fléchette dans le front se trouve bien dans le roman, il n'y est pas mis en valeur comme il l'est dans l'adaptation, où il est un des éléments qui sert au récit filmique à se distancer du récit écrit. Mais c'est par l'isotopie du miroir, d'abord simple outil spéculaire pour ensuite concentrer sur lui la pathologie du personnage, que le film décolle pour prendre son autonomie.

Mais si l'adaptation cinématographique de La Salle de bain parvient à une certaine autonomie vis-à-vis du roman, elle n'en acquiert pas cependant une complète indépendance. Il me semble que le spectateur est sans cesse invité à retourner au texte romanesque par la voix du personnage.

J'ai décidé d'appeler cette voix une voix blanche. La voix du personnage semble désincarnée ; lorsqu'il parle, son visage n'exprime rien. Le personnage semble davantage réciter de manière neutre un texte qui ne le concerne pas, qu'exprimer de 
quelconques sentiments. L'effet ainsi obtenu est très différent d'une voix over (dans ce dernier cas, le locuteur est absent de l'écran).

Ainsi lors d'une averse, le personnage semble moins disserter personnellement sur les différentes manières de regarder tomber la pluie que réciter exactement et d'un ton tout à fait détaché le paragraphe du roman qui relate cet épisode (pp. 35-36). Lorsque, dans le film, il est interrompu par Kabrowinski qui vient lui demander un renseignement, le personnage n'a aucun mal à reprendre exactement là où il l'avait laissé le fil de son discours.

Le discours du personnage dans le film renvoie, par l'effet de «lecture» de cette voix «blanche», au récit écrit.

C'est donc à l'origine par le miroir que le texte filmique, effet spéculaire du texte écrit, s'en décolle pour cependant sans cesse y retourner en nous y renvoyant.

\section{Queen's University}

\section{NOTES}

1 «Histoire» dans le sens donné dans la terminologie de Gérard Genette : «Je propose (...) de nommer histoire le signifié ou contenu narratif (même si ce contenu se trouve être, en l'occurence, d'une faible intensité dramatique ou teneur événementielle)." "J'emploierai encore dans le même sens le terme diégèse, qui nous vient des théoriciens du récit cinématographique.» Figures III (Paris : Seuil, 1972) p. 72, texte et note 1 . L'«histoire» n'est ni le «récit», qui est le texte de l'histoire, ni la narration, qui «est» l'acte d'énonciation qui produit le récit. Effectivement comme le montre le synopsis que j'en donne, le roman et le film sont à faible teneur événementielle.

2 Lors du colloque Écrit/Écran, François Jost a mentionné, dans sa communication intitulée «Le spectateur qui en savait trop», que la caméra subjective est en fait une fausse caméra subjective, c'est-à-dire que, à un moment donné, la caméra nous montre un point de vue que le personnage est techniquement incapable d'avoir à ce moment-là. Ceci nous intéresse, car lors du passage d'un roman à la première personne à l'adaptation cinématographique, comme c'est le cas ici, va se poser le problème de la focalisation (passage de la focalisation interne fixe à ... ?).

3 On reconnaît au passage un emprunt au titre d'un ouvrage de Francis Vanoye, Récit écrit, récit filmique (Paris : Nathan, 1989).

4 Mais en même temps, la mise à distance (avec l'humour entre autres moyens - cf. plus loin) opérée par l'énonciateur fait qu'il semble absent de son propre discours. Marie-Claire Ropars Wuilleumier le signalait à propos du film, le personnage joue un rôle. Nous aurions là en germe une double personnalité, ce qui expliquerait le développement - très évident dans le film - pathologique vers la schizophrénie.

5 Ceci a été éliminé du film. 
6 Ceci pose le problème de la relativité généralisée. Il est remarquable que trois ans après la parution du roman de Jean-Philippe Toussaint, l'astrophysicien britannique Stephen Hawking, dans son best-seller A Brief History of Time - From the Big Bang to Black Holes (New York : Bantam Books, 1988), ait choisi d'illustrer ce phénomène à l'aide d'un exemple relatif au train :

If one carried out experiments with moving bodies on a train, all Newton's laws would still hold. For instance, playing Ping-Pong on the train, one would find that the ball obeyed Newton's laws just like a ball on a table by the track. So there is no way to tell whether it is the train or the earth that is moving.

The lack of an absolute standard of rest meant that one could not determine whether two events that took place at different times occurred in the same position in space. For example, suppose our Ping-Pong ball on the train bounces straight up and down, hitting the table twice on the same spot one second apart. To someone on the track, the two bounces would seem to take place about forty meters apart, because the train would have traveled that far down the track between the bounces. The non existence of absolute rest therefore meant that one could not give an event an absolute position in space, as Aristotle had believed (...).

Both Aristotle and Newton believed in absolute time. That is they believed that one could unambiguously measure the interval of time between two events, and that this time would be the same whoever measured it, provided they used a good clock. Time was completely separate from and independant of space. This is what most people would take to be the commonsense view. However, we had to change our ideas about space and time (pp. 17-18).

Ce rapport espace/temps, qui s'articule sur des problématiques cartésienne et pascalienne et qui fait également référence, au traitement du rapport espace/temps par le philosophe grec Zénon d'Élée (et ses paradoxes de la flèche et d'Achille et la tortue), fait, de ma part, en ce moment, l'objet d'une recherche plus approfondie et ne peut trouver sa place dans l'espace restreint imparti à cette étude, qui n'est qu'un état de recherche en cours.

7 Éliminé de l'adaptation cinématographique.

8 Ceci est gardé dans le film dans une scène qui combine les réflexions sur la perfection de la dame blanche : «Un Mondrian. Le chocolat onctueux sur la vanille glacée, le chaud et le froid, la consistance et la fluidité. Déséquilibre et rigueur, exactitude» (p. 15), et le paragraphe cité qui est prononcé d'une voix que je qualifie plus loin de «blanche» et sur laquelle je vais revenir.

9

The truly modern artist consciously perceives the abstractness of the emotion of beauty : he consciously recognizes aesthetic emotion as cosmic, universal. This conscious recognition results in an abstract creation, directs him toward the purely universal.

That is why the new art cannot be manifested as (naturalistic) concrete representation, which - even when universal vision is present - always points more or less to the particular, or in any case conceals the universal within it.

The new plastic cannot be cloaked by what is characteristic of the particular, natural form and colour, but must be expressed by the abstraction of form and colour - by means of the straight line and determinate primary colour. (...) 
Whereas in nature equilibrated relationship is expressed by position, dimension and value of natural form and colour, in the «abstract» it is expressed through position, dimension and value of the straight line and rectangular (colour) plane.

In nature, we perceive that all relationship is governed by one relationship above all others : That of extreme opposites.

The abstract plastic of relationship expresses this basic relationship determinately - by duality of position, the perpendicular. This relationship of position is the most equilibrated because it expresses the relationship of extreme positions in complete harmony and includes all other relationships. If we see two extremes as manifestations of the inward and the outward, we find that in Neoplastic the bond between spirit and life is unbroken - we see Neoplastic not as denying the full life, but as the reconciliation of the matter- mind duality. (Piet Mondrian, "Neoplasticism in Painting», De Stijl, vol.1 no 1 (1917) pp. 2-6, in Hans L. C. Jaffé, De Stijl (London :

Thames and Hudson, 1970) pp. 36-38.)

Sont éliminées de l'adaptation cinématographique toutes les références directes à Mondrian, mais au début du film, l'on voit le personnage principal arranger soigneusement deux serviettes et deux gants de toilette sur le porte-serviettes. Puis la caméra opère un plan fixe sur le résultat qui rappelle un tableau de Mondrian. Il y a là un parti pris esthétique mais aussi la volonté du personnage d'organiser le monde dans lequel il évolue. Monde équilibré, bientôt déséquilibré par la visite d'un ami de ses parents qui, non content de bouleverser l'arrangement délicat (il va prendre une des serviettes pour s'essuyer les cheveux, car il arrive de l'extérieur où il pleut), va perturber d'un va-et-vient de plus en plus agressif la quiétude du personnage tranquillement allongé dans sa baignoire.

10 Voir note 4.

11 À propos du pronom personnel «il» : « (...) la "3e personne" n'est pas une "personne"; c'est même la forme verbale qui a pour fonction d'exprimer la non-personne». Émile Benveniste, Problèmes de linguistique générale, 1 (Paris : Gallimard, 1966) p. 228. C'est peut-être cette absence d'identité qui fait que dans le film le personnage va passer de la schizothymie à la schizophrénie.

12 En effet, nous dit Michel Serre, la montre ne marque pas le temps directement avec les aiguilles de la trotteuse qui se contentent de le découper en petites unités d'espace. C'est par le mouvement intérieur de l'usure que la montre marque le temps. C'est cette isotopie de l'usure qui renvoie au «mouvement intérieur de mon corps qui se détruisait» (Toussaint, p. 51).

\section{OUVRAGES CITÉS}

«De la plus belle eau». Le Canard enchaîné (mercredi 8 mars 1989).

«Visages pâles». Le Monde (dimanche 12-lundi 13 mars 1989).

Gaudreault, André et Jost, François. Le Récit cinématographique. Paris : Nathan, 1990.

Genette, Gérard. Figures III. Paris : Seuil, 1972.

Grand Larousse de la langue française, 6 vol. Paris : Larousse, 1971.

Pascal. Pensées. Paris : Garnier-Flammarion, 1976.

Toussaint, Jean-Philippe. La Salle de bain. Paris : Minuit, 1985. 